\title{
Disseminating and Sharing Information Through Time-Aware Public Displays
}

\author{
Marcela D. Rodríguez ${ }^{1}$, Angel G. Andrade ${ }^{1}$, Maria Luisa González ${ }^{1}$, \\ and Alberto L. Morán ${ }^{2}$ \\ ${ }^{1}$ Ingeniería en Computación, Facultad de Ingeniería, UABC, Mexicali, B.C., México \\ ${ }^{2}$ Ciencias Computacionales, Facultad de Ciencias, UABC, Ensenada, B.C., México \\ University of Baja California (UABC), Mexicali, México \\ \{marcerod, angel_andrade, alberto_moran\} @uabc.mx, \\ mlglez@yaqui.mxl.uabc.mx
}

\begin{abstract}
Organizations use several methods to communicate formal and informal information. Currently, message boards or corkboards are used as a means for sharing information, coordinating activities and communicating with others. Using our organization as a case study, we observed that there are many message boards around the physical environment and most of them are covered with documents announcing upcoming meetings, seminars, conferences and other events. Besides that, some of the documents were out-of-date which makes it more difficult for users to find information that is relevant for their activities. To support the dissemination of information in organizations, we are proposing a Time-aware Public Display System that enables organization personnel to become aware of relevant information in a timely fashion. We used the Perspective Wall visualization to enable the categorization of the information into topics and dates. We evaluated the system design which shows that users considered appropriate and useful the presentation of information on the Time-aware Public Display.
\end{abstract}

Keywords: Information presentation, Workplace Study, Software Agents.

\section{Introduction}

Organizations use several methods to communicate formal and informal information. These methods can be electronic means, such as e-mails and web pages, and others that depend on the users' location in order for them to get the information. For instance, corkboards are located throughout the physical environment in order to advertise important information for the users' activities. Taking our organization as a case study, a campus of the Autonomous University of Baja California (UABC), we observed that there are many physical corkboards around the campus and most of them are covered with documents announcing upcoming meetings, seminars, conferences and other events. Besides that, some of the documents were out-of-date which makes it more difficult for users to find relevant information for their activities or role (such as students, full professors and research professors). We noticed that publishers on the corkboards use several techniques for attracting the attention of the 
target public. For instance, information considered as urgent and which is used for coordinating activities is presented in documents with a highlighted message. Based on the above observations we identified some issues that should be investigated. For this, we carried out a case study based on the Rapid Contextual Design technique [2]. Based on the results of the case study, we identified the functional requirements of our system and propose a Time-aware Public Display System to support the dissemination of information in organizations by allowing organization personnel to be aware of the information addressed to them. The public display-based system uses the Perspective Wall [1] visualization technique to attract the attention of the target users and enables them to easily locate information that may be relevant for their activities.

Before presenting our solution, we first present in section 2 other related projects, section 3 describes the workplace study we conducted to get a deeper understanding of the sharing and dissemination of information in our organization; section 4 explains the functional requirements of our system; section 5 describes the functionality and architecture of the proposed system; section 6 presents some of the results of the evaluation of the system design; and finally, section 7 presents conclusions and future work.

\section{Related Work}

Other projects have also proposed the use of electronic public displays for sharing information. Notification Collage [4], IM-Here [5] and MessyBoard [6] use public displays for presenting documents that were arbitrarily posted on the public display. As the public display can become saturated with information, it may be difficult for users to be aware of important information. The difference between these projects and The Plasma Poster Network, is that this last system was created not only with the aim of keeping users informed, but to encourage users to initiate conversations. For this, it is recommended that one must "create attractive, inviting interfaces where content changes regularly" [7]. They proposed a plasma display for publishing dynamic multimedia content, which changes every 60 seconds, and enables users to pause the presentation to see the information of his interest. However, even though this technique could be attractive for readers, users must spend time locating information that may be important for them, and when they walk in front of the display they may not be aware of information of their interest.

\section{Case Study}

With the purpose of getting a good and solid understanding of the dissemination of information in organizations by using message boards, we used qualitative methodologies, such as observations and informal interviews. Thus, we got a preliminary understanding of the use of message boards by observing the different boards located throughout our University Campus. We observed that message boards or corkboards are mainly located in the university laboratories. Figure 1 shows a corkboard at the entrance on the Computer Laboratory. We realized that different 
kinds of documents are posted on corkboards located in places where persons usually walk (such as the laboratory entrances and halls) and on the windows of the managers' offices, which very often have to be visited by the organization personnel. We noticed that the same documents can be published in several corkboards; a corkboard contains different types of documents (i.e., for coordinating academic activities, advertising the selling of computers) with information addressed to students and/or professors; and finally, we noticed that some of the published documents were out-of-date. The above preliminary observations may indicate that organization personnel are not opportunistically aware of the information relevant for their activities since for locating and identifying relevant documents they must spend time everyday in order to be aware of new information.

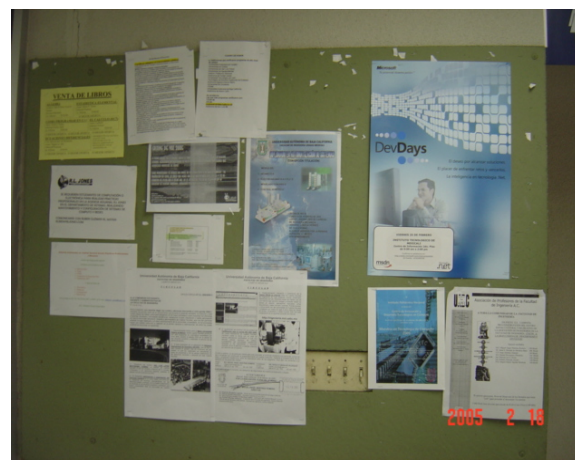

a)

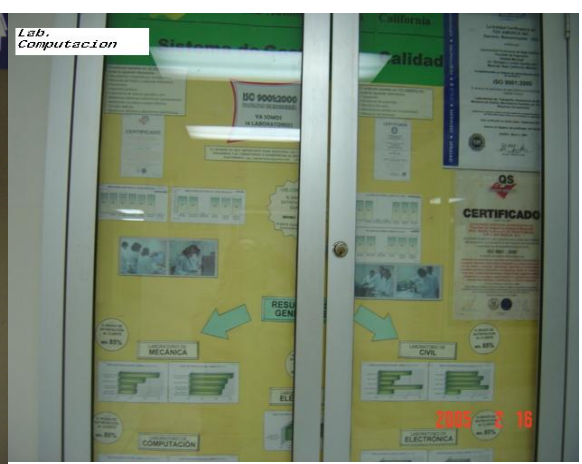

b)

Fig. 1. a) Public message board and b) Private message board located in the hall of the Computer Laboratory

Based on the above observations we identified some issues that should be investigated through our case study. For this, we based our case study on the Rapid Contextual Design technique [2]. We interviewed the organization personnel that frequently publish information (Career Managers and Laboratory Managers that have to coordinate many activities) and those to whom the information is addressed (such as part-time and full-time professors). Figures 2 and 3 show some of the diagrams illustrating the results we obtained from the interviews. The information we collected from our interviewees was categorized by using post-it notes of different colors. Similar answers and comments (which were written on yellow post-it notes) given by the interviewees were categorize by topics (blue post-it notes) that gave us an idea of their practices for publishing or consulting information, and then we grouped the related issues in high level topics (pink post-it notes) that enabled us to identify the requirements of a Public Display-based System for disseminating information.

\subsection{Publishing Practices}

To obtain information regarding publishing practices, we interviewed four (4) Career Managers and four (4) Laboratory Managers who manage the information on the 
corkboards. We asked them questions related to the priorities for publishing, type of information they usually publish, document organization, and information maintenance.

Our findings were grouped into the following categories:

The information is published in several places. Our interviewees agreed that they publish different types of documents (call for meetings, courses for professors, conferences, or news related to academic events). Based on the urgency or importance of the document they decide where to publish the document. Thus, the most relevant information is published in several places around campus, and in some places from which this information can not be removed by anyone, such as the windows of the Managers' offices.

There are priorities for publishing information. We found that for publishing information, message boards can be classified as public, semi-public and private. Any person (professors, managers, students, and others who may not be personnel of the organization such as sellers) can publish or remove any document from the public message boards. For publishing on the semi-public message boards, users are required to ask for permission from the Managers. These boards contain exclusively academic information and information used for coordinating the personnel activities. And finally, only the Managers can publish information on the private message boards. The private message boards, as shown in figure $1 \mathrm{~b}$ ), have information that due to its relevance, should remain for a period of time. For instance, it may contain information related to the rules for using laboratory equipment.

There are priorities for removing documents from the message boards. We found that only managers have authorization for removing documents from private and semipublic boards. From public boards, any person can remove a document, i.e. when there is no space for posting a document, the publisher removes an out-of-date document, or posts it on top of an expired document. As public boards can become saturated with documents, they are cleaned at the end of each course period (semester).

Different types of information are published by managers. They must make information available that is relevant for the organization personnel, such as academic events; and information relevant for coordinating their activities, such as upcoming meetings. Thus, message boards are used by managers for communicating with the organization personnel.

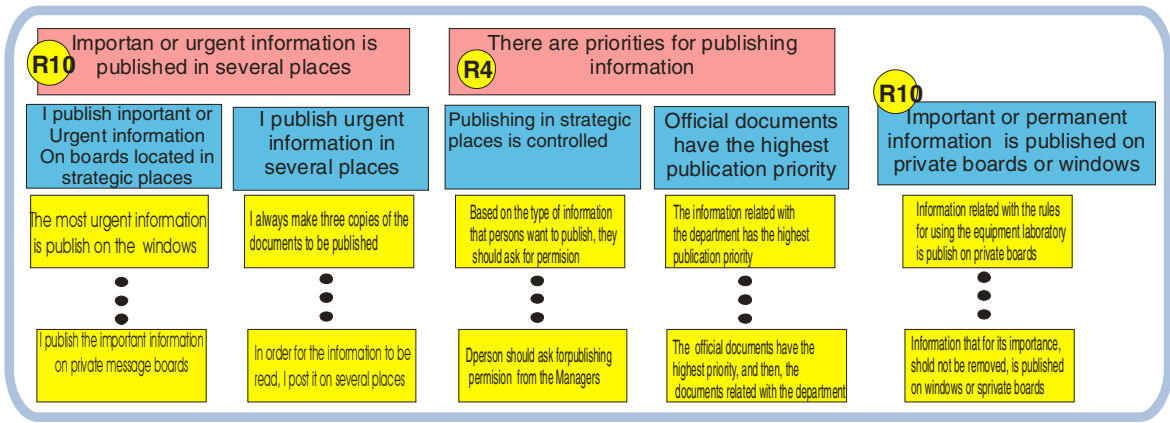

Fig. 2. Findings from the interviews for finding out the publishing practices 


\subsection{Consulting Practices}

We also interviewed eight (8) professors. We asked them about when and where they look for information, what usually attracts their attention, and about the relevance of the information they find on the message boards. We ask them to tell us about the last time they consulted a corkboard, and the last time they found information of interest to them. This stage of the case study enabled us to mainly identify the different types of information that the subjects considered more important for their activities and define the categories in which the information can be classified (see figure 3). We also found out how our informants locate and keep the important information. These findings are described next.

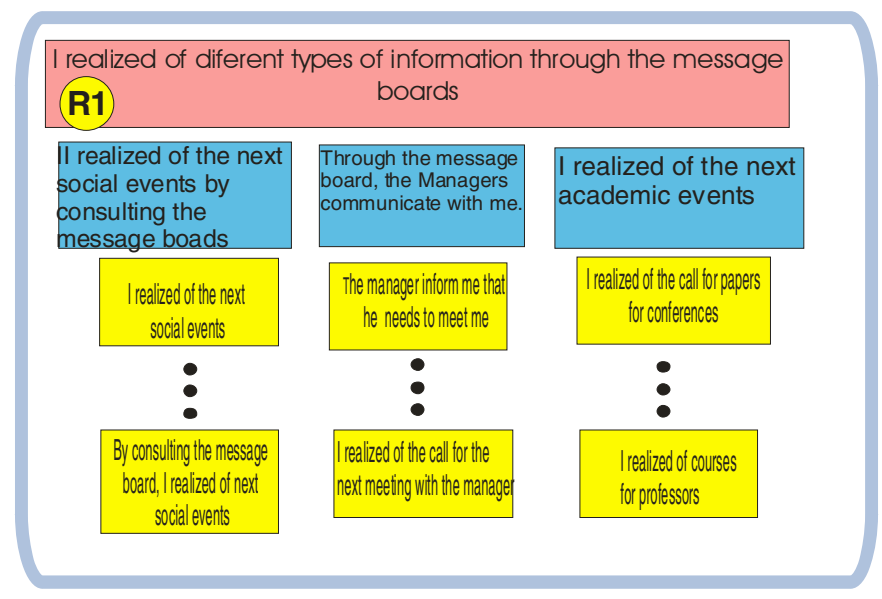

Fig. 3. Findings from the interviews for finding out the consulting practices

Message boards are used to communicate different types of information. The interviewees stated that they usually get information about upcoming academic events such as conferences and courses, social and cultural events from the public message boards. They also stated that these boards are used by managers for communicating with them, i.e. professors are notified of upcoming meetings.

Sometimes users did not view important messages in a timely fashion. The informants stated that they usually approach the boards when a document visually attracts their attention, or sometimes they explicitly approach the board for finding important information. However, for some of them, consulting the message boards is not a constant activity and they tend to miss important information.

Users keep the important information with them. Some of the interviewees said that they make note of the most important information contained in a document, for instance dates of an event or meeting, URL of the document. 


\section{Functional Requirements}

From the results of our case study, we identified the following requirements of a public-display based system that enables organization personnel to be aware of the information addressed to them.

The public display system must be time-aware. The system must automatically remove out-of-date documents and be aware of upcoming deadlines to publish reminders. Thus, the system will enable users to not get distracted by the documents that are not relevant anymore.

Public displays should be located in places that are the most visited by the users. Our findings indicated that information should be pervasive to reach the users. To do this, several public displays should be located throughout the physical environment and in strategic places in the organization to keep personnel informed.

The system should provide mechanisms for authorizing users to publish or modifying information. The public display-based system should provide mechanisms for deciding who has permission for publishing or modifying the content of each of the public displays available in the environment.

The system should facilitate the publishing of different types of documents from the users' computer. The public display system should facilitate the publication of different types of information which may be contained in documents of different formats, such as images, text documents, or web files.

The information should be classified in categories. We identified four main categories in which documents can be easily located by users: Academic Events (i.e. Conferences, Courses for professors), Notifications (i.e. notifications of upcoming meeting), Social and Cultural Events, and News (news related to the University Campus).

\section{Time-Aware Public Display System}

Our solution uses the Perspective Wall visualization technique in order to enable users to easily find the information relevant for their activities. This technique enables the presentation of information which is classified in topics, and it also emphasizes the information that is currently important. This technique has the advantage that it requires the peripheral attention from users. Thus, users may decide to approach the public display to consult a document. In the following sections we explain the design of the user interface based on this visualization technique and then we present the design of the system architecture.

\subsection{Design of the User Interface}

The public display is divided into three perspectives: left, center and right as illustrated in Figure 4. The center and right perspectives have vertical axes which define three categories (Academic Events, Notifications, and Social and Cultural Events) and horizontal axes that indicate the time in which the information is relevant. 
For instance, a Call for papers for a Conference will always appear in the first row (Academic Events) and the column of the current week (July 9-15). This document will also appear in the first column of the right perspective with a notification attached to it. This notification will indicate to the users that there is a deadline on July 30-31. This type of document will appear on the row of the current week until the deadline is reached. The left perspective of the Wall presents dynamic content of recent news of the organization. Finally, the left perspective also enables users to visualize more information about any of the documents they select from the center or right perspectives.

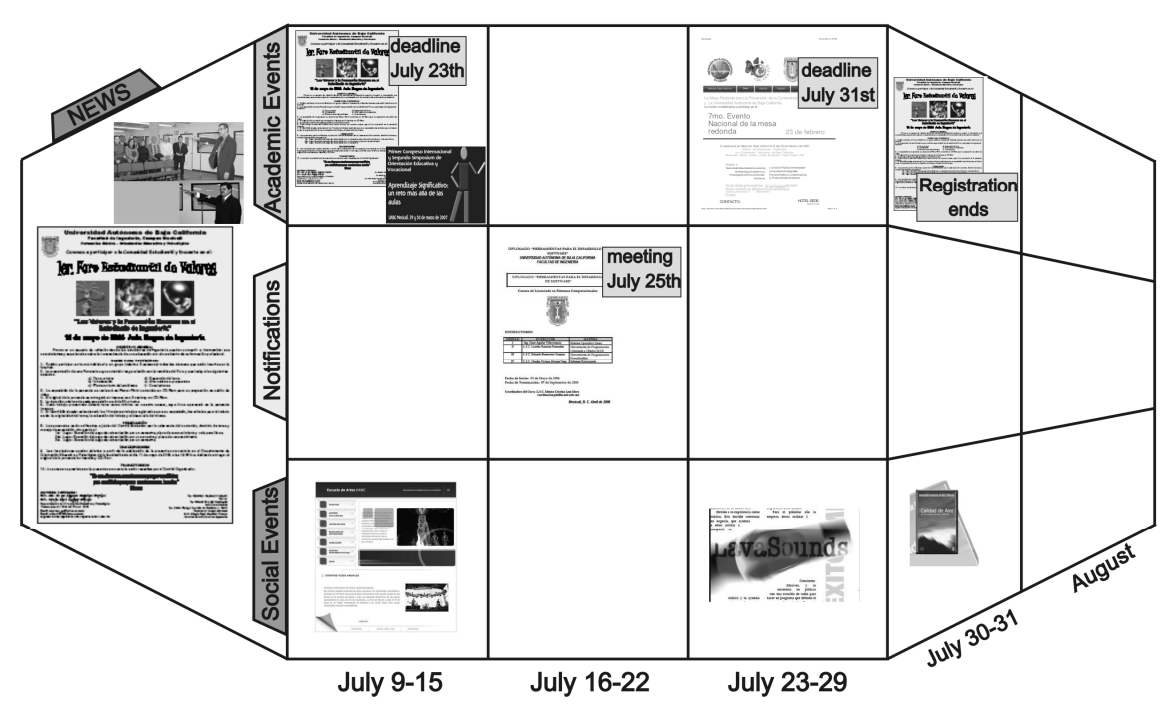

Fig. 4. Time-aware public display

\subsection{Architecture of the System}

Figure 5 shows that the system consists of several nodes executing components. Some of the system's components were identified as software agents that may act as proxy to a device, or carry out a functionality that must be hidden to the user. These agents autonomously decide how to act based on the information they perceived [3]. The system's nodes and components are the following:

Display Server Node. This is a node containing the components that publish, remove or modify the information presented on the Public Display. It contains a Web Server which enables any authorized user to publish information from any Client with a Web Browser.

Web Server Node. It contains the components for enabling the user to upload a document to the public-display with are the following:

- Submission Form which is a web form that the users can invoke from any Client with a Web browser. The Submitted Form requests the document to be published and 
other information related to the document (meta-information) such as the category, the deadline, the URL of the web page in which there is more information related to the document, and the identity of the person who posts the document.

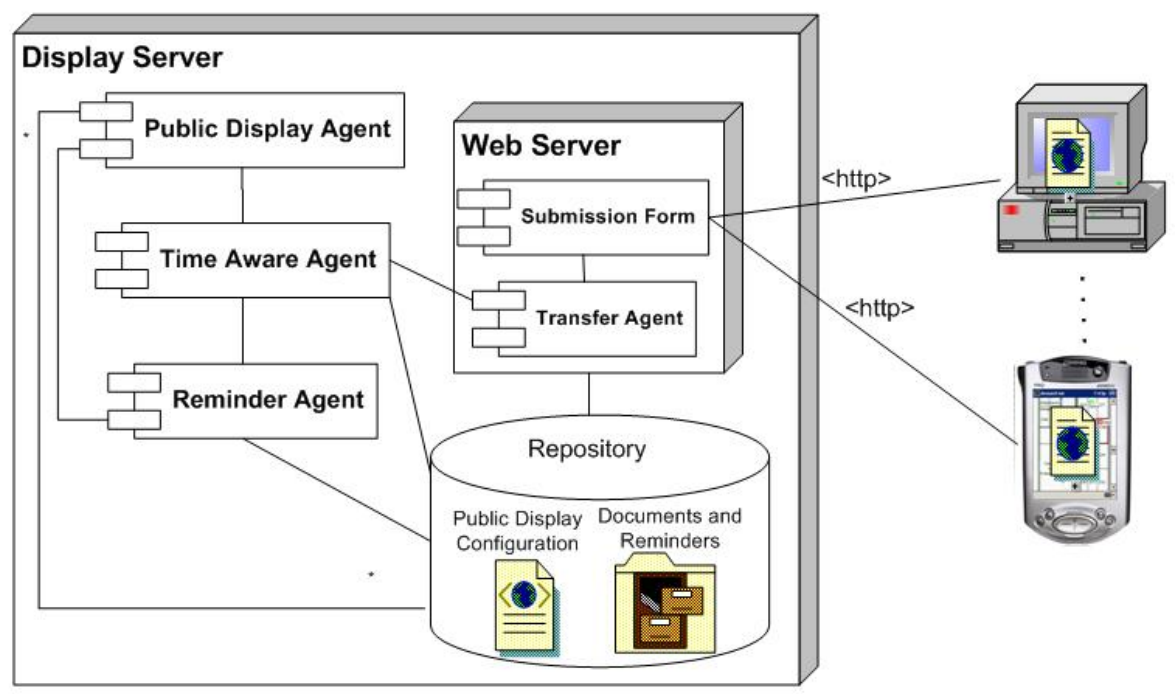

Fig. 5. Architecture of the Time-aware Public Display System

- Transfer Agent. This sends the document to the Repository. It also gets the metainformation introduced by the user, and transforms it into an XML format which is delivered to the Time-aware Agent and added to the Public Display Configuration of the Repository component.

Time-aware Agent. It analyzes the document's meta-information in order to identify where to publish the new document (category and date). Then, the Timeaware Agent notifies the Public Display Agent that a new document has to be posted. The Time-aware Agent will be checking when a new document has expired to notify it to the Public Display Agent, and will be aware of documents deadlines in order to ask the Reminder Agent to create a reminder.

Reminder Agent. This agent creates reminders about deadlines. When a new reminder is created, the Reminder Agent saves it in the Repository component and modifies the Public Display Configuration. Then, the Reminder Agent informs the Public Display Agent that there is a new reminder to be published.

Public Display Agent. This agent acts as a proxy to the public display by publishing or removing information from the public display. The Public Display Agent is informed by the Time-aware Agent or the Reminder Agent if there is a new document or reminder to be published which have to be retrieved from the Repository component by the Public Display Agent. 


\section{Evaluation of the System Design}

We have already evaluated the system design with the aim of knowing the users' perception of ease of use and utility of our technological solution. For participating in the evaluation we selected 20 professors. The evaluation consisted in a 2 hours session in which: i) we first found out how and when they consult the organization's message boards, and what type of information they mainly consult; ii) we presented two scenarios of use to illustrate the functionality of the Time-aware Public Display System; iii) finally, we asked questions to know the opinion of the participants with respect to the utility of the system and the facility to locate information relevant for their activities.

All the participants (20) said that they approach a message board at least once a week. Three (3) participants stated they approach a message board looking for information that may be relevant for them, thirteen (13) participants stated that they approach a message board only when a document attracts their attention and four (4) participants mentioned the above two reasons. The information most consulted was the documents posted by Managers and the Principal of the Engineering School (such as notifications of upcoming meetings), following by information about Conferences and Symposiums, and the information less consulted was related with Cultural and Social Events.

The evaluation results of the design of the Time-aware Public Display System showed that the participants considered useful the system. One of the participants commented that: "as the documents are not posted in all message boards of the organization, sometimes we do not read important information,... the system will enable us to notice when there is important information"; other participant stated: "the system will enable us to organize information, and therefore it will also facilitate us to locate important information". Finally, all the participants considered appropriate to visualize the information categorized (by topics and dates), and that the reminders will enable them to be opportunistically aware of important dates.

\section{Conclusions and Future Work}

In this paper we propose to support the dissemination and sharing of information in organizations through a Time-aware Public Display System by enabling the organization personnel to be opportunistically aware of the information addressed to them. The evaluation results showed that currently the message boards are mainly used for communicated information from Managers to the organization personnel. Thus, the public display-based system will enable this communication carries out efficiently by enabling the organization personnel to be aware of important information opportunistically. An important system's characteristic is the Perspective Wall [1] visualization technique to attract the attention of users, which requires the peripheral attention from users in order for them to realize that there is new information that may be important for them.

We are planning to incorporate mechanisms that enable users to transfer the information published, to their personal information space. For this, we are envisioning that users can transfer the relevant information they found on the Public Display by using their mobile phones or Personal Digital Assistants. 


\section{Acknowledgements}

We thank the UABC professors that participated in the case study and the evaluation session. This work was funded by UABC and CONACYT under contract J51582-R.

\section{References}

[1] Mackinlay, J.D., Robertson, G.G., Card, S.K.: The Perspective Wall and Context Smootly Integrated. ACM Conference on Human Factors in Computing System (CHI), pp. 174-179 (1999)

[2] Holtzblatt, K., Burns-Wendell, J., Wood, S.: Rapid Contextual Design (Ed), p. 316. Elsevier, New York (2005)

[3] Rodriguez, M.D., Favela, J., Preciado, A., Vizcaino, A.: Agent-based ambient intelligence for healthcare. In: AI Communications, vol. 18(3), pp. 201-216. IOS Press, Amsterdam (2005)

[4] Greenberg, S., Rounding, M.: The Notification Collage. ACM Conference on Human Factors in Computing Systems (CHI), pp. 514-521 (2001)

[5] Huang, E.M., Russel, D.M., Sue, A.E.: IM Here: Public Instant Messaging on Large, Shared Displays for Workgroup Interactions. ACM Conference on Human Factors in Computing Systems (CHI), pp. 279-286 (2004)

[6] Fass, A., Forlizzi, J., Paush, R.: MessyDesk and MessyBoard: two Designs Inspired by the Goal of Improving Human Memory. DIS, pp. 303-311 (2002)

[7] Churchill, E.F., Nelson, L., Denoue, L., Girgensohn, A.: The Plasma Poster Network: Posting Multimedia Content in Public Places. NTERACT, Ninth IFIP TC13 International Conference on Human-Computer Interaction (2003) 